\title{
Armenian Astronomy: Recent Activities
}

\author{
A. M. Mickaelian, S. V. Farmanyan
}

Address: NAS RA V. Ambartsumian Byurakan Astrophysical Observatory (BAO)

E-mail:aregmick@yahoo.com

\begin{abstract}
An overview of the current activities of the Armenian astronomy, including professional research, University and school education, public outreach and other organizational affairs. We show that present day Armenian astronomy serves as a leader for the regional activities, as well as is among the most advanced and active sciences in Armenia. Moreover, recent achievements during the years 2000-2018 are such that many years efforts since Viktor Ambartsumian's times were necessary and finally received recognition in various fields. BAO was recognized as National Value, regional astronomical centre, and Markarian Survey was included in UNESCO Memory of the World documentary heritage list. We succeeded to organize a number of important meetings (IAU symposia and colloquium, JENAM, etc.) and establish a series of Byurakan International Summer Schools. We also present the Armenian Astronomical Society's (ArAS) and IAU South West and Central Asian regional centre's activities.
\end{abstract}

\section{Introduction}

NAS RA V. Ambartsumian Byurakan Astrophysical Observatory (BAO) is one of the most important astronomical centres in the territory of Former Soviet Union (FSU) and in the Middle East region. It was founded in 1946 by the great Armenian scientist Viktor Ambartsumian (1908-1996), who in fact initiated and determined the main research directions of the Observatory, namely mostly connected to the instability phenomena in the Universe, at both stellar and galactic levels. The discovery of stellar associations in 1947 and the hypothesis on the activity of the galactic nuclei in 19541955 by V. Ambartsumian were the two main directions to focus on by many other BAO astronomers. Another great Armenian astronomer Beniamin Markarian (19131985 ) with his colleagues, based on V. Ambartsumian's ideas accomplished in 19651980 one of the most important astronomical surveys of the $20^{\text {th }}$ century, the First Byurakan Survey (FBS or Markarian Survey), which resulted in the discovery of 1500 UV-excess (UVX) galaxies (Markarian galaxies). Many other important investigations were carried out by Grigor Gurzadyan, Marat Arakelian, Romela Shahbazian, Ludwik Mirzoyan, Edward Khachikian, Elma Parsamian and others, as well as by the theoreticians Arthur Nikogossian, Norair Yengibaryan, Mamikon Mnatsakanian and 
others resulting in the discovery of dozens of thousands of new cosmic objects and a number of important astrophysical results. A large international project for search and studies of flare stars was carried out by L. Mirzoyan and colleagues and resulted in the discovery of thousands of flare and T Tauri stars in stellar associations and young stellar clusters. The Second Byurakan Survey (SBS) was carried out by B. Markarian, Jivan Stepanian and others in 1978-1991 and resulted in the discovery of some 3600 new objects, both galaxies and peculiar stars. The $2^{\text {nd }}$ part of the FBS carried out by Hamlet Abrahamian, Areg Mickaelian and Kamo Gigoyan resulted in the discovery of some 2500 UVX and red stars and blue stellar objects (QSOs and Seyfert galaxies). A. Mickaelian also accomplished optical identifications of IR and Xray sources based on spectroscopic surveys (Byurakan and Hamburg) and revealed thousands of new active galaxies and peculiar stars. He also accomplished the digitization project of the FBS in 2002-2007 and created the largest Armenian astronomical database DFBS, which is available for the astronomical community. Armen Gyulbudaghian, Tigran Magakian and colleagues revealed many new young stellar objects important for understanding of the stellar evolution.

Beside the top-level research, Armenian astronomers have initiated and accomplished many other activities and always have maintained the Armenian astronomy in the frontiers of the world astronomy. 6 IAU symposia and colloquia were organized in Armenia in 1966, 1986, 1989, 1998, 2001 and 2013. The Armenian Astronomical Society (ArAS) was established in 2001 and joined the European Astronomical Society (EAS) as an affiliated member. The European Annual Astronomical Meeting (JENAM) was organized in Armenia in 2007, the largest ever scientific event in Armenia. Based on the DFBS, the Armenian Virtual Observatory (ArVO) was created in 2005 and joined the International Virtual Observatory Alliance (IVOA). In 2006, ArAS and BAO initiated the series of the Byurakan International Summer Schools (BISS), one every two years and one of them, the 3BISS was combined with the IAU International School for Young Astronomers (ISYA) in 2010. 6 such schools have been organized so far. In 2011, the DFBS was included in the UNESCO "Memory of the World" International register of documentary heritage, a rare case for scientific items. In 2013, BAO was recognized by the Armenian Government as National Value. Since 2015, Armenia hosts one of the IAU Regional Offices of Astronomy for Development (ROAD), namely the South West and Central Asian (SWCA) one. Since 2017 Armenia joined the European journal Astronomy \& Astrophysics, one of the top-level astronomical journals in the world. Armenian astronomers also run a lot of educational and outreach activities; an excellent example is the very successful participation of Armenian school pupils to the 
International Astronomical Olympiads since 1996. In this paper we give a general understanding of current activities of the Armenian astronomy.

\section{Observing facilities and projects}

At present BAO has several medium and small telescopes: $2.6 \mathrm{~m}$ classical reflector, $1 \mathrm{~m}$ Schmidt, 0.5m Schmidt, and several 0.4-0.5 m size telescopes. Detailed description of the big telescopes is given at BAO and ArAS webpages. SCORPIO focal reducer is the main instrument used for photometry and long-slit spectroscopy, as well as VAGR multi-pupil spectrograph and Speckle-interferometer (guest instrument from Spain, University of Santiago de Compostela) are available. A number of interesting observing projects are being accomplished with BAO telescopes, including some international ones. Here we list the current observing projects accepted for the first half of 2019.

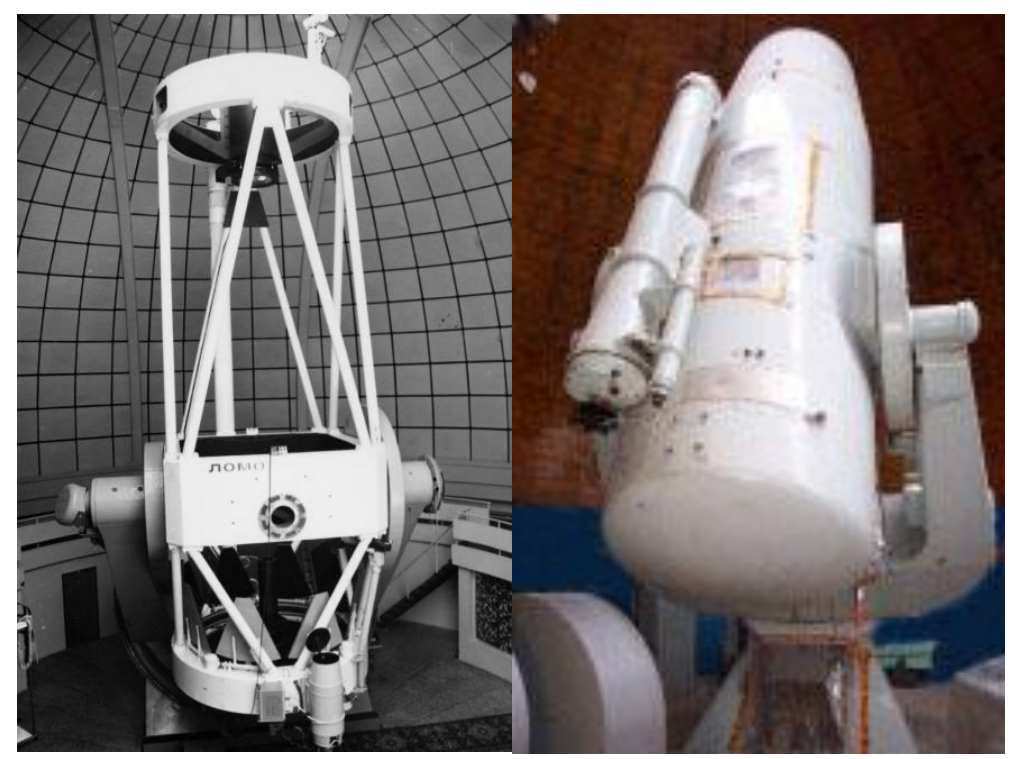

\section{6 m telescope}

Abrahamyan $\mathrm{H}$. (BAO, Armenia) - Spectral study of radio variable sources

Dennefeld M. (IAP, France) - Classification of transients

Gigoyan K. (BAO, Armenia) - Search and spectroscopic study of the Catalina surveys periodic variables

Hakopian S. (BAO, Armenia) - Star-forming regions in galaxies

Krugly Y. (IAKKNU, Ukraine) - The study of the influence of non-gravitational effects on the rotation of asteroids in results of photometric observations

Magakian T. (BAO, Armenia) - Eruptive young stars 
Melikian N., Docobo J. (BAO, Armenia, OARMA, Spain) - Speckle interferometry of close binaries

Miroshnichenko A. (UNCG, USA) - The largest group of stars with the $B[e]$ phenomenon

Pozanenko A. (IKI, Russia) - Gamma-ray burst follow up observations

Wyrzykowski L. (WUAO, Poland) - Black holes in the Milky Way and beyond from Gaia transients

\section{1 m Schmidt telescope}

Movsessian T. (BAO), Dodonov S. (SAO, Russia) - Search for distant quasars by means of multiband photometry

\section{Research Departments}

BAO current structure was established in 201710 research departments are active that include many important astrophysical and astronomical subjects:

- Astronomical Surveys (Head: Dr. A. M. Mickaelian)

- Non-Stable Phenomena (Head: Dr. H. A. Harutyunian)

- Young Stellar Objects (Head: Dr. T. Yu. Magakian)

- Astrochemistry, Astrobiology and Exoplanets (Head: Dr. A. G. Yeghikyan)

- Active Galactic Nuclei (Head: Dr. R. R. Andreasyan)

- Supernovae (Head: Dr. A. A. Hakobyan)

- Theoretical Astrophysics (Head: Prof. A. G. Nikogossian)

- High-Energy Astrophysics (Head: Dr. G. T. Ter-Kazarian)

- Compact Cosmic Objects and Relativistic Gravity (Head: Prof. A. D. Sedrakyan)

- Historical-Cultural Astronomy (Head: Dr. G. H. Brutian)

Most of these subjects are among the traditional BAO research directions (Astronomical Surveys, Non-Stable Phenomena, Young Stellar Objects, Active Galactic Nuclei, Theoretical Astrophysics), however, modern topics are also active (Astrochemistry, Astrobiology and Exoplanets, Supernovae, High-Energy Astrophysics), as well as we encourage the development of Historical-Cultural Astronomy to link our studies to humanities and culture.

In addition, 3 infrastructural departments were created:

- Observational Astronomy 
- Astroinformatics

- Applied Astronomy

Especially important is the development of Astroinformatics to keep our research at international top level. We describe in the next section the creation and development of this department and related activities.

\section{Astroinformatics}

Along with research departments, Astroinformatics infrastructural department was established in 2017 in BAO. It is created to develop and coordinate the following areas: BAO Local Area Network (LAN), BAO Internet, BAO webpage, BAO Plate Archive Project, ArVO, the collaboration with NAS RA Institute of Informatics and Automation Problems (IIAP), scientific computational tasks, including:

- astrostatistical calculations

- mathematical modeling,

- cross-correlations of catalogs and large databases,

- graphical presentations and visualization,

- animations,

- simulations (by development of new research ideas),

- VO developments,

- Laboratory Astrophysics,

- otherscientific and technical tasks.

\section{International Collaboration}

BAO has always been very active in international collaboration. During many decades, BAO maintains tight contact and collaboration with a number of research centres and other scientific organizations. Especially active is the collaboration with France, Germany, Italy, Russia, USA and Spain. We list here all countries and institutions having collaboration with BAO and Armenian Astronomy:

Chile - Universidad de Chile

China - National Astronomical Observatories, Chinese Acad. Sci., Beijing 
France - IAP, Obs. Paris-Meudon(OBSPM), Obs. Marseille, Obs. Haute-Provence (OHP), Univ. Montpellier II, Obs. Lyon (CRAL), LATMOS, IMCCE, Obs. Grenoble, CDS Georgia - Abastumani Astrophysical Observatory(AbAO); IAU SWCA ROAD Germany - ARI (Heidelberg), AIP (Potsdam), Hamburger Sternwarte (HS), Frankfurt University, Jena

Iran - IPM, Astronomical Society; IAU SWCA ROAD

Italy - Univerista di Roma "La Sapienza", Padova Astronomical Obs., Asiago, ICRANet Jordan - AUASS, Al Al-Bayt University, Mafraq; IAU ROADs

Kazakhstan - FIAp; IAU SWCA ROAD

Mexico - Tonantzintla Observatory, INAOE (Puebla), UNAM

Netherlands - Leiden; IAU ROADs

Poland - Warsaw University

Portugal- CAUP

Russia - Special Astrophysical Obs. (SAO), IKI, SPbSU, SPbPU, INASAN, SAIMSU

Spain - University of Santiago de Compostela, IAC

Tajikistan - IAp; IAU SWCA ROAD

Turkey - Astronomical Society; IAU SWCA ROAD

UK - UCLAN (Preston), DIAS, Armagh Observatory

USA - Cornell University, Space Telescope Science Institute(STScl)

\section{International Meetings and Schools}

IAU symposia and colloquia have been the most important meetings in astronomy. Every observatory and astronomical institute consider their organization as an honour and as a prestigious event. BAO has organized 6 such meetings:

$\begin{array}{lll}1966 & \text { IAU S029 } & \text { Non-Stable Phenomena in Galaxies } \\ 1986 & \text { IAU S129 } & \text { Observational Evidence of Activity in Galaxies } \\ 1989 & \text { IAU S137 } & \text { Flare stars in Star Clusters, Associations and Solar Vicinity } \\ 1998 & \text { IAU S194 } & \text { Activity in Galaxies and Related Phenomena } \\ 2001 & \text { IAU C184 } & \text { AGN Surveys } \\ 2013 & \text { IAU S304 } & \text { Multiwavelength AGN Surveys and Studies }\end{array}$

Another important meeting, the largest ever scientific event in Armenia was organized jointly by EAS, ArAS and BAO in 2007, the Joint European and National Astronomical Meeting (JENAM-2007). At present such meetings are called EWASS 
(European Week of Astronomy and Space Science) and are being organized every year in one of the European countries.

The first international meeting on the problem of Extraterrestrial Intelligence was organized in BAO in 1971 (CETI 1971). Competent scientists, working in various fields, concerning the considered complex problem, were present at the symposium: astronomers, physicists, radiophysicists, cyberneticists, biologists, chemists, archaeologists, linguists, anthropologists, historians, and sociologists. 32 Soviet and 19 American scientists, as well as representatives from UK, Hungary, and Czechoslovakia participated in the works of the symposium. Three Nobel Prize winners were among the participants: biologist F. Crick (UK) and physicists F.W. Dyson and C. Towns (USA).

We organize many other meetings, including joint ones (ByurakanAbastumani Colloquia, Armenian-French Workshops, and Armenian-Iranian Workshop), Anniversary Meetings.

Among the educational activities the most important is the series of the Byurakan International Summer Schools (BISS), one of which (3BISS) was combined with the IAU International School for Young Astronomers (ISYA-2010). Byurakan Summer Schools for YSU students (BSS) are to select students for future involvement in BAO. BAO Science Camps (BSC) are for school pupils and serve for attraction of young generation to astronomy.

\section{BAO International Integration}

Here we give the list of the Armenian Astronomy activities related to international integration during the recent years (2000-2018).

$2001 \quad$ IAU Colloquium \#184: AGN Surveyswas organized in Byurakan.

$2001 \quad$ ArAS foundation and affiliation to EAS. Currently it has 100 members.

2002-2007 Digitized First Byurakan Survey (DFBS, Digitized Markarian Survey). 1874 photographic plates were digitized and electronic database was created.

2005 Armenian Virtual Observatory (ArVO) was created and joined the International Virtual Observatory Alliance (IVOA).

2006 Byurakan International Summer Schools (BISS) series were founded. BISS are being held every two years and are considered as one of the top=level schools in the world . 
2007 JENAM-2007: Our non-stable Universewas organized in Yerevan State University (YSU). The biggest ever scientific event in Armenia.

20103 3BISS / IAU ISYA-2010. The $3^{\text {rd }}$ BISS was combined with the IAU International School for Young Astronomers (ISYA) and was one of the biggest such events in the history of ISYAs.

2010 V. Ambartsumian International Science Prize was established; International Steering Committee was created. The first award to Michel Mayor and his team.

2011 Markarian Survey and DFBS in UNESCO“Memory of the World"(MOW) International Register. It is one of the rarest scientific items in all UNESCO lists.

2013 BAO was awarded by RA Government a status of RA National Value. International support letters played a crucial role.

2013 Armenia became an associated member of the International Planetary Data Alliance (IPDA).

2013 BAO became an associated member of ICSU World Data System (ICSU WDS).

2013 ICRANet Armenian Branch was established in Yerevan. It is one of the institutes of NAS RA.

2013 IAU Symposium \#304: Multiwavelength AGN Surveys and Studies was organized in NAS RA, Yerevan. The biggest ever IAU symposium organized in Armenia.

2014 Horizon 2020 (H2020) Space coordination by BAO scientists.

2014 BAOInternational Science Advisory Committee (ISAC)was created for the first time to revise BAO activities and to give recommendations for its further development.

2014 Armenian Space Agency (ArSA) was created in frame of UN COPUOS program.

2014 IAU WG on Archaeoastronomy and Astronomy in Culture (AAC) was created and Armenian astronomers took an active part in it.

2015 IAU South West Asian Regional Office of Astronomy for Development (SWAROAD)was established in Byurakan, Armenia. In 2016 after the affiliation of Kazakhstan and Tajikistan it was renamed to IAU South West and Central Asian (SWCA) ROAD. In 2017, Turkey also joined IAU SWCA ROAD.

2015-2018 BAO Plate Archive Project (Digitization and Scientific Usage) was accomplished. 

was organized at NAS RA, Yerevan. Astrophysics (A\&A).

We see that Armenian astronomers are at least not less active than before, during the high-level funding at the Soviet times.

\section{Armenian Astronomical Society (ArAS)}

The Armenian Astronomical Society (ArAS) was in fact created on 22 June 1999, when the first database of Armenian astronomers was collected, it was decided to create the society, By-Laws were developed and ArAS founding meeting was held. It is true that the Society was officially registered by the Armenian Ministry of Justice in two years, on 29 August 2001. Anyway, before that actions were taken to involve astronomers in the membership, identify and organize the future activities of ArAS. After the official registration, the first steps were the affiliation to the European Astronomical Society (EAS) in September 2001, the creation of ArAS website in 2002, preparation and distribution of electronic newsletters ("ArASNews") since 2002 and establishment of the ArAS annual meetings since 2002. Since then, almost all events in Armenian astronomy are to a large extent connected with ArAS, and in the 2000s Armenian astronomy by its activeness has reached and in some way exceeded the previous successes present during the Soviet Union years.

\section{Membership}

ArAS is an organization of exclusively professional astronomers (though a few exceptions are allowed), and also aims at establishing close ties with the Armenian foreign astronomers, inviting them to join and cooperate with astronomers in Armenia. ArAS founding members were 16 astronomers of the Byurakan Astrophysical Observatory (BAO). ArAS currently has 100 members (including 14 founding ones) representing 49 institutions from 21 countries (http://www.aras.am/Members/members.html), including: Armenia - 50 (BAO - 29, YSU - 6 and others), USA - 15, Germany - 6, Russia - 5, France - 4, Mexico - 3, Italy and Spain - 2 (each), Bulgaria, Canada, Chile, Greece, Hungary, India, Ireland, Netherlands, Portugal, Romania, Switzerland, Thailand, and UK - 1 (each). Among the famous ArAS members there are Michel Mayor (Switzerland), Brent Tully (USA), Vahe 
Petrosian (USA), James Houck (USA), Daniel Weedman (USA), Yervant Terzian (USA), Igor Karachentsev (Russia), Zadig Mouradian (France), Daniel Kunth (France), Massimo Turatto (Italy) and many others. We have created for each member a personal webpage containing basic information on his personal and professional data. ArAS has 3 Co-Presidents: Haik Harutyunian, Areg Mickaelian (Acting President), and Yervant Terzian, ArAS Vice-President: Tigran Magakian, ArAS Scientific Secretary: Elena Nikoghosyan, Treasurer: Marietta Gyulzadyan, ArASNews Editor: Sona Farmanyan, and ArAS Webpage Administrator: Gor Mikayelyan.

\section{ArAS Electronic Newsletter}

Until recently ArAS released periodically electronic newsletters (ArAS Newsletters, ArASNews (http://www.aras.am/ArasNews/arasnews.html) typically 8 times in a year (periodicity of 1.5 month). The Editors have been: Tigran Magakian (2002-2004, issues \#1-12), Lusine Sargsyan (2005, issues \#13-16), Lilit Hovhannisyan (2006-2007, issues \#17-24), Areg Mickaelian (2008-2014, issues \#25-76) and Sona Farmanyan is the editor since 2015 (issues \#77-124). Since 2015 the Newsletter became monthly, 12 issues in a year. In total, including 2017, 112 issues have been prepared and released. In total, in all ArASNews, already 929 articles have been published with total 1495 pages. ArAS Newsletters give news, announcements, articles on Armenian astronomy and BAO, ArAS new members, international and local meetings, summer/winter schools and participation of Armenian astronomers in them, astronomical education in Armenia, Armenian archaeoastronomy and astronomy in culture, anniversaries, scientific, scientific-popular, information materials, etc. A Reference List (http://www.aras.am/ArasNews/arasnewsreference.html) of ArASNews articles has been also created allowing easy access to all articles related to any subject.

\section{ArAS webpage}

ArAS webpage (http://www.aras.am/) was created in February 2002, at first to give the necessary information about ArAS, including ArAS objectives and forms of activities, list of ArAS members, annual meetings and other events, etc. In early 2009, in connection with the International Year of Astronomy (IYA-2009), ArAS webpage was fundamentally enriched and renewed and became a repository containing full information on Armenian astronomy. There is information about BAO history, achievements, current projects and international collaboration, received grants, all publications since 2000 have been installed, all meetings with individual pages, new sections have been opened for other scientific institutions in Armenia related to 
astronomy, 21 famous Armenian astronomers, database of 257 Armenian astronomers throughout the world, astronomical education, amateur astronomy, archaeoastronomy, etc. Without exaggeration, in terms of the amount of information, ArAS webpage is the richest one among all Armenian scientific organizations and one of the best in the world. We also published in ArAS website Calendars of Astronomical Events of 2014-2018.

\section{Participation in international organizations}

Armenia is one of 73 member-states of the International Astronomical Union (IAU), as well as ArAS is recognized by IAU. Areg Mickaelian is the Secretary and the Acting President of the IAU National Committee for Astronomy (NCA) in Armenia. ArAS is one of EAS 25 affiliated societies, and by its activities it seconds only the most powerful countries in Europe: UK, Germany, France, Italy and some others. ArAS is also the official representative of the Euro-Asian Astronomical Society (EAAS) in Armenia. Areg Mickaelian is one of the EAAS Vice-Chairs and is a member of the EAAS International Bureau, and Tigran Magakian is a member of the EAAS ScientificTechnical Committee. Together with the Ministry of Education and Science, ArAS is also one of the official representatives of International Astronomical Olympiads (IAO) in Armenia. Armenia joined the international Galileo Teachers Training Program (GTTP) with its official representatives Areg Mickaelian (Ambassador in Armenia) and Marietta Gyulzadyan (Armenian Coordinator). Armenia is an associate member of the Sub-Regional European Astronomical Committee (SREAC) acting in Southeast Europe and an associate member of the International Planetary Data Alliance (IPDA). EAS Annual Meeting (European Week of Astronomy and Space Science, EWASS) was held in April 2018 in Liverpool (UK); ArAS Acting President Areg Mickaelian and other Armenian astronomers took part from Armenia.

\section{ArAS awards}

ArAS has 5 kinds of awards given at ArAS webpage (http://www.aras.am/Prize/awards.htm):

- ArAS Annual Prize for Young Astronomers (Yervant Terzian Prize)

- ArAS/BAO Awards (2009)

- ArAS Certificates

- ArAS/OxArm (Oxford Armenian Society) Awards for scientific journalists

- Galileo Teachers Training Program (GTTP) Certificates 
Since 2004, ArAS awards Annul Prize for Young Astronomers (since 2009 it was renamed to Yervant Terzian Prize). It is being awarded to the most active young astronomers (younger 35) taking into account all annual activities. The award is sponsored by ArAS Co-President Prof. Yervant Terzian and BAO. At present it totals USD 500, and it is being awarded with ArAS Certificate.

\title{
ArAS meetings and other events
}

Since 2002, ArAS holds regular annual meetings, however some of them were associated or combined with other conferences and events, where ArAS acted as coorganizer.

\author{
Year No. ArAS annual meeting description and other related conferences \\ 2002 I Scientific Sessions and Annual Report \\ 2003 II Scientific Sessions combined with Armenian-Georgian Colloquium \\ 2004 III Scientific Sessions and Annual Report \\ 2005 IV Scientific Sessions and Annual Report \\ $2006 \mathrm{~V} \quad$ Scientific Sessions combined with BAO 60 $0^{\text {th }}$ Anniversary Meeting \\ $2007 \mathrm{VI} \quad$ Scientific Sessions combined with $15^{\text {th }}$ EAS Annual Meeting (JENAM- \\ 2007) \\ 2008 VII Scientific Sessions combined with Ambartsumian's $100^{\text {th }}$ \\ Anniversary Meeting \\ 2009 VIII Scientific Sessions related to IYA-2009 (including Astrobiology \\ and Archaeoastronomy) \\ 2010 IX Scientific Sessions and Annual Report \\ 2011 X Scientific Sessions and Annual Report \\ 2012 XI Scientific Sessions combined with Anania Shirakatsi 1400 meeting \\ 2013 XII Annual Report and Summary of Astronomical Year 2013 \\ 2014 XIII Scientific Sessions combined with RASCS meeting \\ 2015 XIV Scientific Sessions combined with Armenian-Iranian Astronomical \\ Workshop \\ 2016 XV Annual Report and Summary of Astronomical Year 2016 \\ 2017 XVI Sci. Sessions combined with the Int. Conf. "Astronomical Heritage of the \\ Middle East"
}

ArAS XIII annual meeting was unprecedented in its theme. It took place on 710 October 2014 in Byurakan and was called "Relation of Astronomy to Other 
$\begin{array}{llll}\text { Sciences, } & \text { Culture } & \text { Society" }\end{array}$ http://www.aras.am/Meetings/RASCS/index.html). In addition to astrophysical issues it included Philosophical Problems of Astronomy, Astrobiology, Astroinformatics, Astronautics, Archaeoastronomy, Astronomical Education, Amateur Astronomy, Scientific Tourism and other topics related to astronomy. It brought together astronomers, philosophers, historians, archeologists, philologists, artists and representatives of other fields. ArAS dedicated a few small-scale events (one-day conferences or seminars) to the anniversaries of famous astronomers.

\section{Summer Schools and Science Camps}

Since 2006, ArAS and BAO organize regular Byurakan International Summer Schools (BISS,http://www.aras.am/Meetings/meetingsSummerSchools.html), once every two years. Since 2005, local summer schools for YSU Physics Department students are being organized to cause and increase interest to astronomy. They are the continuation of the initiative started in 1995. ArAS has also organized a Conference for Young Astronomers in 2011.

\section{Year Summer school name, Short}

$20051^{\text {st }}$ Byurakan Summer School for YSU PhysDep students, 1BSS

$20061^{\text {st }}$ Byurakan International Summer School, 1BISS

$20082^{\text {nd }}$ Byurakan International Summer School, 2BISS

$20092^{\text {nd }}$ Byurakan Summer School for YSU PhysDep students, 2BSS

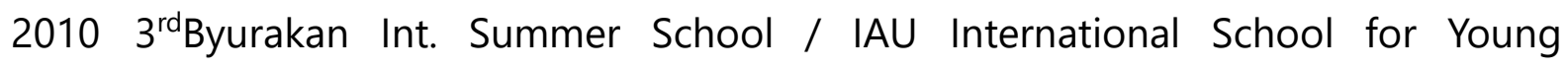
Astronomers, 3BISS/ISYA

2011 FSU countries Young Scientists Conference, FSU-YSC

$20124^{\text {th }}$ Byurakan International Summer School, 4BISS

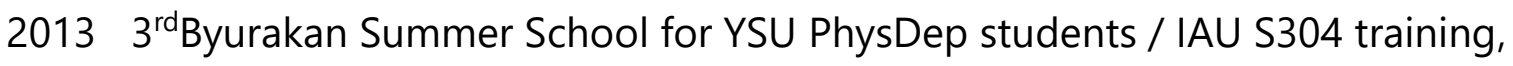
3BSS

$20165^{\text {th }}$ Byurakan International Summer School, 5BISS

$20186^{\text {th }}$ Byurakan International Summer School, 6BISS

Armenian young astronomers regularly attend several international schools and conferences: IAU International Schools for Young Astronomers (ISYA), European NEON/OPTICON schools, Vatican Observatory Summer Schools (VOSS), Virtual Observatory (VO) training schools, and a number of others. ArAS website holds a directory table of the most important regular international 
schools\footnote\{\ur|\{http://www.aras.am/SS2010/ss_other_schools.htm\}\} which helps young astronomers and students to follow and find their respective schools based on the level of the students, location, subject, etc.

Since 2014, on the initiative of Sona Farmanyan, ArAS also organizes Byurakan Science Camps (BSC) for school pupils aged 12-15. Fund for Armenian Relief (FAR) partially sponsors these camps. In 2018, the Fifth Byurakan Science Camp was organized.

\section{Astronomical Olympiads}

Since 1996, International Astronomical Olympiads (IAO) and since 2007, International Olympiads on Astronomy and Astrophysics (IAOO) are being organized. Armenian pupils have regularly taken part in both of them and have achieved excellent results. In total, they have won 9 Gold, 5 Silver and 20 Bronze medals. Marietta Gyulzadyan has especially big contribution in the achievements of our team; she has been the team leader since 2006. Among the most successful participants are: Zhirayr Avetisyan and Mkrtich Soghomonyan (each have won one I, one II, and one III rank prizes), Tigran Shahverdyan (one II and one III rank prizes), Tigran Nazaryan, Hayk Saribekyan, Hayk Tepanyan, Hayk Hakobyan and Edgar Vardanyan have one I rank prize each. Igor Chilingarian from Russia has won I rank prize as well.

\section{ArAS School Lectures}

ArAS Co-President Yervant Terzian and some other American sponsors of Armenian origin, co-finance a program of ArAS School Lectures, which runs jointly with the Armenian Ministry of Education and Science (MES). The lecturers are professional astronomers and their visits leave vivid impression for the school children. Schools are also given astronomical materials (books, booklets, sky maps, calendars, etc.), connection is being established with gifted children. In 2012, 2013, 2014, 2016 and 2018 such a program was implemented in Armenia and Artsakh schools.

\section{Other matters of astronomical education}

ArAS tries to contribute to the astronomical education matters in Armenia. Summer Schools, Science Camps and Olympiads are also in this field. Other activities include installation of various information on ArAS webpage, preparation and release of educational CD/DVDs (recently released "Astronomy for schools" and "Astronomy for students" DVDs, which are collections of astronomical textbooks, books, dictionaries, encyclopedias, articles, reports, photos, videos, software, etc.). In November 2014, on the initiative of Sona Farmanyan ArAS founded Facebook group "Junior Astronomer's 
Club" (JAC). "Viktor Ambartsumian's Descendants" Educational Charitable Foundation was founded in August 2014. Yervant Terzian is the Chair of its Board of Trustees, Areg Mickaelian is its Coordinator, and Robert Sargsyan is the Director of the Foundation.

\section{Archaeoastronomy and Astronomy in Culture}

To coordinate issues related to Archaeoastronomy, an appropriate section was opened ArAS webpage (http://www.aras.am/Archaeoastronomy/astronomyancientarmenia.html). These questions are currently given great importance in the world, in particular, they are in the focus of international organizations such as UNESCO ("Astronomy and World Heritage" project), IAU (Working Group "Astronomy and World Heritage"), International Council of Monuments and Sites (ICOMOS), European Society for Astronomy in Culture (Societe Europeen ne pour l'Astronomie dans la culture, SEAC), "Starlight" initiative and others. In 2014, IAU created a new working group "Archaeoastronomy and Astronomy in Culture" (AAC). Already several related meetings have been organized in Armenia in 2011, 2012, 2014, 2015, 2016 and 2017. ArAS Newsletter now has a permanent section "Archaeoastronomy and Astronomy in Culture".

\section{Astronomy outreach}

Armenia actively participates in the IAU Astronomy for Development (we have established a regional office in Armenia; see the corresponding article) and Astronomy Outreach programs. The Armenian Coordinator of the latter is Sona Farmanyan.

ArAS gives importance to the development of amateur astronomy. There is "Goodricke John" amateur astronomers organization created by Ruben Buniatyan, which put efforts and reached the recognition of 18 September (Viktor Ambartsumian's birthday) as Astronomy Day in Armenia. ArAS created on its webpage a section for amateur astronomy (further to establish Armenian Amateur Astronomical Society, ArAAS), as well as a Facebook group, where one can register as an amateur astronomer.

In December 2010, to promote scientific, especially astronomical journalism, the group of Scientific Journalists in Armenia was formed, which includes more than 100 journalists. ArAS periodically prepares and disseminates press releases, organizes 
press conferences, interviews, and scientific journalism seminars. Press releases contain astronomical news, events organized by ArAS and BAO and held in BAO, occurred and expected celestial phenomena, as well as scientists anniversaries, Armenian and other international scientific news. Astronomers hold public lectures at different organizations. On ArAS webpage, online "Astghagitak" ("Astronomy Expert") popular astronomical journal was created, where materials in Armenian are being placed for those interested in astronomy.

ArAS publishes a number of books (Proceedings of its Annual Meetings), popular booklets, calendars, sky maps and other promotional material. 